\title{
SOME UNCOMPLEMENTED FUNCTION ALGEBRAS
}

\author{
BY \\ I. GLICKSBERG(1)
}

1. Let $X$ be the unit circle $|z|=1$, and $A$ the "disc algebra" of functions on $X$ having continuous extensions to $|z| \leqq 1$ analytic for $|z|<1$. Then it is known [12] that there is no bounded projection of $C(X)$ onto $A$; alternatively, $A$ is uncomplemented in $C(X)$.

To what extent is this a general occurrence? Specifically, if $A$ is a closed nonselfadjoint $\left({ }^{2}\right)$ subalgebra of $C(X), X$ compact, is $A$ uncomplemented in $C(X)$ ?

Only some partial results will emerge here. From recent results of Bishop [1], extended to the nonmetric case by Bishop and deLeeuw in [2], we obtain the curious fact that if $X$ is the Šilov boundary of $A$, and $T$ is any bounded operator on $C(X)$ acting as the identity on $A$, then $\|T-I\|=\|T\|-1$, where $I$ is the identity operator; alternatively, any operator $S(=I-T)$ annihilating $A$ has $\|I+S\|=1+\|S\|$. As a consequence of this fact one can apply the technique of [12] to show that if $X$ is a compact group and our nonselfadjoint subalgebra $A$ of $C(X)$ is translation invariant, $A$ is uncomplemented; in fact any closed subspace lying between two invariant algebras $A_{1} \subset A_{2}$, with the set of conjugates $\bar{A}_{1} \notin A_{2}$, is uncomplemented ( $\S 3$ ). And this applies equally well to invariant subalgebras $A_{1}, A_{2}$ of $\left({ }^{3}\right) C_{0}(X)$, where $X$ is a locally compact abelian group ( $\left.\$ 4\right)$; but both proofs are technically complicated, and shed no light on the situation in general.

In what follows we shall consider a slightly more general setting in which $A$ is a subalgebra of $C_{0}(X), X$ locally compact; since we shall be concerned with estimating the norms of projections, the usual adjunction of an identity does not lead easily to a reduction to the compact case.

The author is indebted to K. deLeeuw for several helpful comments and, in particular, Theorem 4.1 is due to deLeeuw.

2. Let $X$ be locally compact and the Šilov boundary of $A$, a closed subalgebra of $C_{0}(X)$, so $A$ separates the points of $X$. We shall denote by $\mathscr{P}$ the set of all $x$

Presented to the Society, January 26, 1963; received by the editors February 4, 1963.

(1) Work supported in part by the National Science Foundation through Grant G-22052 and in part by the Air Force Office of Scientific Research.

(2) There are simple examples of complemented selfadjoint subalgebras; e.g., see Corollary 3.2.

(3) $C_{0}(X)$ denotes the space of continuous functions vanishing at infinity on the locally compact space $X$ (which is identical with $C(X)$ if $X$ is compact). 
in $X$ for which, for every neighborhood $V$ of $x$, there is an $f_{V}$ in $A$ which is identically 1 on a subset of $V$ containing $x$, and elsewhere has modulus $<1$, i.e., $x \in f_{V}^{-1}(1) \subset V$ and $\left|f_{V}\right|<1$ off $f_{V}^{-1}(1)$. It is a consequence of results of Bishop and deLeeuw $[2,6.5]$ (more directly obtainable from the earlier arguments of Bishop [1], as in [11, 3.3.15]) that every element of $A$ assumes its maximum modulus over $X$ on $\mathscr{P}$ ( $=$ the set of Condition II points of [2], or strong boundary points of [11]), whence $\mathscr{P}$ - contains the Šilov boundary of $A$, and $\left({ }^{4}\right) \mathscr{P}$ is dense in the Šilov boundary $X$ of $A$.

Now suppose $B$ is a closed subspace of $C_{0}(X)$ which is also an $A$-module $(A B \subset B)$. Then for any (finite complex regular Borel) measure $\mu$ on $X$ orthogonal to $B$

$$
\mu\{x\}=0
$$

for any $x$ in $\mathscr{P}$ which is not a common zero of $B$. For with $V$ any neighborhood of $X$ and $f=f_{V}$ in $A$ as above, $f^{n} \mu$, the usual product of function and measure, is orthogonal to $B$ since $A B \subset B$; thus (by dominated convergence) for $b$ in $B$ we have

$$
0=\int b f^{n} d \mu \rightarrow \int_{f^{-1}(1)} b d \mu,
$$

and, by the regularity of $\mu$ and continuity of $b, b(x) \mu\{x\}=0$, whence the assertion.

Now these two properties of $\mathscr{P}$ yield the following fact, which is fundamental to our considerations.

LEMMA 2.1. Let $X$ be the Šilov boundary of the closed subalgebra $A$ of $C_{0}(X)$, and let $B \subset C_{0}(X)$ be a closed A-module whose common zeroes form $a$ nowhere dense subset of $X$. Let $F$ be a closed subspace of $C_{0}(X)$ containing $B$, and $T: F \rightarrow C_{0}(X)$ a bounded linear transformation. If

$$
T b=b, \text { all } b \text { in } B,
$$

then

$$
\|T-I\|=\|T\|-1
$$

where $I$ is the identity operator.

(Here $\|T-I\|$ is computed over the domain $F$ of $T-I$.) In particular, if $F \neq B$, any bounded projection $T$ of $F$ onto $B$ has norm $\geqq 2$; for $T$ cannot be invertible in the algebra of bounded operators on $F$, so $1 \leqq\|T-I\| \leqq\|T\|-1$.

(4) Actually the results of $[2 ; 11]$ are given only for an algebra (containing the constants) on a compact space. But the results extend by the standard procedure: imbed our $A$ as a maximal ideal in such a subalgebra $A_{\infty}$ of $C\left(X_{\infty}\right), X_{\infty}$ the one point compactification of $X$. Then for any Condition II point [2] or strong boundary point [11] $x$ of $A_{\infty}$ in $X$ and for any neighborhood $V$ of $x$, with $\infty \notin V$, we have an $f$ in $A_{\infty}$ with $x \in f^{-1}(1) \subset V,|f|<1$ off $f^{-1}(1)$, so $|f(\infty)|<1$. If $\phi$ is a conformal self map of $|z| \leqq 1$ onto itself with $\phi(f(\infty))=0, \phi(1)=1$, then $\phi \circ f \in A_{\infty}$ (since $\phi$ is analytic near the spectrum of $f$ ) and $\phi \circ f(\infty)=0$. Thus $\phi \circ f \in A$, and $x \in \mathscr{P}$. Since such $x$ (along with $\infty$ ) are dense in $X_{\infty}[2 ; 11], \mathscr{P}$ is dense in $X$. 
Proof of 2.1. Let $x \in X$. Then $f \rightarrow T f(x)$ is a linear functional on $F$ of norm $\leqq\|T\|$. By the Hahn-Banach theorem and the Riesz representation theorem we have a measure $\lambda_{x}$ on $X$ of norm $\leqq\|T\|$ with

$$
T f(x)=\int f d \lambda_{x}, \quad f \in F .
$$

Let $\mu_{x}$ be the unit point mass at $x$, and set $v_{x}=\mu_{x}-\lambda_{x}$, so

$$
f(x)-T f(x)=\int f d v_{x}, \quad f \in F .
$$

Since $T$ acts as the identity on $B, v_{x}$ is orthogonal to $B$, and so $v_{x}\{y\}=0$ if $y \in \mathscr{P}$ and $B(y) \neq 0$. In particular if $x \in \mathscr{P}$ and $B(x) \neq 0, v_{x}\{x\}=0$ and $v_{x}$ and $\mu_{x}$ are mutually singular; hence

$$
\left\|v_{x}\right\|+1=\left\|v_{x}\right\|+\left\|\mu_{x}\right\|=\left\|\lambda_{x}\right\| \leqq\|T\|,
$$

and $\left\|v_{x}\right\| \leqq\|T\|-1$. By (2.1) we thus have

$$
|T f(x)-f(x)| \leqq\|f\|(\|T\|-1)
$$

for each $x$ in $\mathscr{P}$ which is not a common zero of $B$. But $\mathscr{P}$ is dense in $X$ while the common zeroes of $B$ are nowhere dense, so (2.2) holds for a dense subset of $X$, and

$$
\|T f-f\| \leqq\|f\|(\|T\|-1), \quad\|T-I\| \leqq\|T\|-1 .
$$

The reverse inequality is trivial, and 2.1 is proved.

One situation (which we shall meet again) in which 2.1 yields quite simply the nonexistence of certain bounded projections is that of the following:

Corollary 2.2. Let $A_{1}, A_{2}$ be closed subalgebras of $C_{0}(X)$ having $X$ as their Šlov boundaries, and let $B_{i} \subset C_{0}(X)$ be a closed $A_{i}$-module whose common zeroes are nowhere dense in $X, i=1,2$, with $B_{1} \cap B_{2}=0$. Then no bounded operator $T$ can act as the identity on $B_{1}$ and also annihilate $B_{2}$.

For otherwise $T$, acting as the identity on $B_{1}$, satisfies $\|T-I\|=\|T\|-1$, while $I-T$ acts as the identity on $B_{2}$, so that

$$
\|T\|=\|(I-T)-I\|=\|I-T\|-1=\|T\|-2 .
$$

Note that 2.2 says that although (by hypothesis) the sum $B_{1}+B_{2}$ is direct we cannot enlarge each $B_{i}$ to a closed subspace $C_{i}$ so that the sum $C_{1}+C_{2}$ is direct and closed in $C_{0}(X)$.

Remark 2.3. If $f \in C_{0}(X) \backslash B$ and $F=C f+B, C=$ complexes, then it is well known (and easily proved) that for every $\varepsilon>0$ there is a projection of $F$ onto $B$ of norm $<2+\varepsilon$. The fact that all such projections are of norm $\geqq 2$ for our $B$ of 2.1 represents a curious geometric aspect of the imbedding of $B$ into $C_{0}(X)$, which can easily be rephrased as follows, where ball $C_{0}(X)$ is the unit ball in $C_{0}(X)$ : The subset 


$$
B \cap\left(C f+\text { ball } C_{0}(X)\right)
$$

of $C_{0}(X)$ has diameter $\geqq 4$ for each $f$ outside $B$.

REMARK 2.4. There can, of course, be subspaces $F \subset B$ and projections $T$ of $F$ onto $B$ of norm precisely 2; for example if $F$ is the "disc algebra," and $A=B$ is the subalgebra of elements $f$ whose analytic extensions to $|z|<1$ have $f^{\prime}(0)=0$, then the projection which we might write as

$$
f(z)=\sum_{n=0}^{\infty} a_{n} z^{n} \rightarrow \sum_{n \neq 1} a_{n} z^{n}
$$

is of norm 2 since $\left|a_{1}\right| \leqq\|f\|=\sup \left|f\left(e^{i \theta}\right)\right|$. However, this is not the case if $F=C(X)(5.1)$.

Remark 2.5. Finally, note that $X$ being the Silov boundary of $A$ is essential to 2.1 and not just to our proof. Indeed let $X$ be the disc $|z| \leqq 1, A$ the "disc algebra" viewed as a subset of $C(X)$ : the subalgebra of all $f$ analytic on $|z|<1$. Let $F$ be the closed subspace of $C(X)$ formed by all $f$ in $C(X)$ with $f\left|T^{1} \in A\right| T^{1}$, $T^{1}=\{z:|z|=1\}$. Alternatively $F=F_{0} \oplus A$, where $F_{0}=\left\{f \in C(X): f\left(T^{1}\right)=0\right\}$, and the natural projection $f_{0}+a \rightarrow a$ is clearly of norm 1 , since

$$
\left\|f_{0}+a\right\| \geqq \sup _{z \in T^{1}}\left|f_{0}(z)+a(z)\right|=\sup _{z \in T^{1}}|a(z)|=\sup _{|z| \leqq 1}|a(z)|=\|a\| .
$$

Nevertheless there is no bounded projection of all of $C(X)$ onto $A$. Indeed for a fairly wide class of algebras once it is known that $A$ is uncomplemented in $C(X)$ for $X$ the Šilov boundary of $A$, then $A$ is uncomplemented in any $C(X)$ into which it can be imbedded. Specifically this holds whenever each multiplicative linear functional on $A$ has a unique (non-negative) representing measure carried by the Šilov boundary $X$ (as in the case of the disc algebra).

For suppose $P$ is a bounded projection of $C(Y)$ onto $A$ (so that $A$ is closed in $C(Y))$ and $B$ is the subalgebra of $C(Y)$ of functions constant on the sets of constancy of $A$; then $B=C(Z)$, where $Z$ is a factor space of $Y$, and we may view $A$ as a closed (separating) subalgebra of $C(Z)$, while $P$ projects $C(Z)$ boundedly onto $A$. We can now view $Z$ as a subspace of the maximal ideal space of $A$, and $Z$ contains the Šilov boundary $X$ of $A$ since $A$ is closed in $C(Z)$. Thus for each $z \in Z$ we have a unique representing measure $\mu_{z}$ carried by $X$ (so $f(z)=\int_{X} f d \mu_{z}$, $f \in A)$. From the uniqueness of $\mu_{z}$ we conclude that $z \rightarrow \mu_{z}$ is weak* continuous into the space of measures on $X$, the dual of $C(X)$, or more precisely, into the weak* compact set $\Sigma$ of normalized non-negative measures on $X$ (for if $z_{\delta} \rightarrow z$ then any weak* cluster point $\mu \in \Sigma$ of $\left\{\mu_{z_{\delta}}\right\}$ satisfies $f(z)=\int_{X} f d \mu, f \in A$, so $\mu=\mu_{z}$ and $\mu_{z_{s}} \rightarrow \mu_{z}$ by the compactness of $\Sigma$ ).

Consequently for $g \in C(X)$, setting

$$
f(z)=\int_{X} g d \mu_{z}, \quad z \in Z,
$$


defines an element $f$ of $C(Z)$, so the subspace $E=\left\{f \in C(Z): f(z)=\int_{X} f d \mu_{z}\right.$, $z \in Z\}$ of $C(Z)$ is (isometrically) isomorphic to $C(X)$. Since $P$ provides a bounded projection of $E$ onto $A$, there must be one of $C(X)$ onto $A$, despite our hypothesis that nome exist.

3. Let $X$ again be the unit circle, and $A$ the "disc algebra"'. The fact that there is no bounded projection of $C(X)$ onto $A$ was found by W. Rudin [12] and, independently, by R. Arens and P. C. Curtis, with both proofs following the lines of [12], and utilizing the group structure of $X$ and the rotation invariance of $A$ (to produce from a bounded projection another commuting with translations). The approach is as follows:

Let $R_{x}$ denote the translation operator: $R_{x} f(y)=f(y x), f \in C(X)$. Then the existence of $P$ implies that

$$
T f=\int R_{x-1} P R_{x} f d x, \quad f \in C(X),
$$

where integration is with respect to normalized Haar measure, defines a bounded linear operator $T$ on $C(X)$. (Since $x \rightarrow R_{x^{-1}} P R_{x} f$ is easily seen to be strongly continuous, the integral can be viewed as strongly convergent.) Moreover one clearly has $T R_{x}=R_{x} T$ and $T f=f$ for $f$ in $A$ : thus for a character $\chi$ of $X$, $T \chi(x)=R_{x} T \chi(1)=T R_{x} \chi(1)=T \chi(x) \chi(1)=\chi(x) T \chi(1)$, and $T \chi=(T \chi(1)) \chi$. So $T \chi=\chi, \chi \in A, T \chi=0, \chi \notin A$. Consequently $T$ coincides with the natural "projection" sending a Fourier series into its "non-negative half" which one knows (by elementary means or, in fact, by 2.2) to be unbounded (or not into $A$ ) yielding the desired contradiction.

This final step is of course the only point at which $X$ need be the circle group, rather than any compact abelian group, with $A$ any translation invariant nonselfadjoint subalgebra of $C(X)$ (where one might apply deeper results, as indicated in [12]). But in fact for such algebras and groups the same result holds as an easy consequence of the introduction of $T$ and the special case, or, rather the final step of its proof; and in fact something more follows:

(3.2) Let $G$ be a compact abelian group. A a closed translation invariant subalgebra of $C(G)$, and $B \subset C(G)$ a closed invariant A-module containing $A$ but not $A$ (so $A$ is not selfadjoint). Then any closed subspace of $C(G)$ lying between $A$ and $B$ is uncomplemented in $C(G)$.

For suppose $P$ is a bounded projection onto such a subspace, and we form $T$ as in (3.1); we so obtain a bounded operator commuting with translations, acting as the identity on $A$, and with range in $B$ (since this was true of $P$ and $B$ is invariant). Let $G^{\wedge}$ be the character group of $G$. As before $T \hat{g}=(T \hat{g}(1)) \cdot \hat{g}, \hat{g} \in G$, so $T \hat{g}=\hat{g}, \hat{g} \in A, T \hat{g}=0, g \notin B$.

As an invariant subspace, $A$ is spanned by the set of characters it contains, $S=A \cap G^{\wedge}$, a subsemigroup of $G^{\wedge}$. Since $B$ in an $A$-module, $S B \subset B$. From 
$\bar{A} \notin B$ there is some $\hat{g} \in A$ with $\hat{g}^{-1}=\overline{\hat{g}} \notin B$, and since $\hat{g}^{-1} \notin S B \subset B$, $\hat{g}^{-1} S^{-1} \cap B=\varnothing$. Thus $\hat{g}^{n} \in A, n \geqq 0$ and $\hat{g}^{n} \notin B, n<0$, whence $T \hat{g}^{n}=\hat{g}^{n}$, $n \geqq 0, T \hat{g}^{n}=0, n<0$, and $\sigma: n \rightarrow \hat{g}^{n}$ is $1-1$.

Dual to the homomorphism $\sigma$ we have another, $\rho: G \rightarrow X$ (the circle group), which has dense range since $\sigma$ is $1-1$, hence is onto since the range is compact. So $X$ is isomorphic to a factor group $G / H$ of $G, H$ the kernel of $\rho$, and $\tilde{\rho} C(X)$, the range of the induced isometry $\tilde{\rho}: f \rightarrow f \circ \rho$ of $C(X)$ into $C(G)$, consists of just those $f$ in $C(G)$ constant on cosets mod $H$. Because $T$ commutes with translations we thus have $T \tilde{\rho} C(X) \subset \tilde{\rho} C(X)$, whence $\tilde{\rho}^{-1} T \tilde{\rho}$ is a bounded operator on $C(X)$. But the character $n$ of $X$ has $\tilde{\rho} n=n^{\circ} \rho=\sigma n=\hat{g}^{n}$, and since $T \hat{g}^{n}$ is $\hat{g}^{n}$ for $n \geqq 0$ and 0 for $n<0, \tilde{\rho}^{-1} T \tilde{\rho}$ preserves (resp., annihilates) the characters of $X$ corresponding to non-negative (resp., negative) integers; we know no bounded operator on $C(X)$ does this, whence the assertion.

The preceding argument is of course restricted to abelian $G$, but the result, or almost all of it, continues to hold for $G$ nonabelian, with 2.2 providing the means for the final step.

THEOREM 3.1. Let $G$ be a compact group, $A_{1} \subset A_{2}$ a pair of translation invariant $\left({ }^{5}\right)$ closed subalgebras of $C(G)$ with $\bar{A}_{1} \notin A_{2}$. Then any closed subspace $B$ of $C(G)$ lying between $A_{1}$ and $A_{2}$ is uncomplemented in $C(G)$.

Proof. Suppose $P$ is a bounded projection of $C(G)$ onto $B$, and form

$$
T_{1} f=\int R_{x^{-1}} P R_{x} f d x, \quad f \in C(G),
$$

so obtaining a bounded operator on $C(G)$ which commutes with right translations, acts as the identity on $A_{1} \subset B$, and has range in $A_{2}$ as before. Again form

$$
T f=\int L_{x-1} T_{1} L_{x} f d x, f \in C(G) ;
$$

since $L_{x} R_{y}=R_{y} L_{x}$ we now have an operator $T$ with all the cited properties of $T_{1}$ which commutes with right and left translations. We shall use this operator to obtain our contradiction.

Now if $A_{1}$ separates the points of $G$, so that $G$ can be viewed as a subspace of the maximal ideal space of $A_{1}$, then $G$ coincides with the Silov boundary of $A_{1}$ (for $G$ contains the Šlov boundary, which thus is a nonvoid subset invariant under translations since $A_{1}$ is). Consequently we shall proceed by first reducing our considerations to the case in which $A_{1}$ separates, and then showing there is a nonzero invariant $\bar{A}_{1}$-module $M$ which $T$ annihilates. This will essentially complete our proof, since then $A_{1}$ and $M$ will be, respectively, $A_{1}$ - and $\bar{A}_{1}$-modules, each without common zeroes on $G$ (since the set of common zeroes, being in-

(5) That is, $R_{x} f$ and $L_{x} f$ are in $A$ for $f$ in $A$, where $L_{x} f(y)=f\left(x^{-1} y\right)$. 
variant, is either void or all of $G$ ), while $G$ is the Šilov boundary of $A_{1}$ and $\bar{A}_{1}$; thus 2.2 will apply to yield our contradiction.

Suppose then that $A_{1}$ does not separate the points of $G$, and partition $G$ into the (closed, disjoint) sets of constancy of $A_{1}$. Since $A_{1}$ is invariant any one of these maps, under a (right or left) translation, into, hence onto, another, and all may be obtained as translates of the set of constancy $H$ containing the identity 1 of $G$. Indeed if $x \in H$ then for the same reason $1 \in x^{-1} H$ implies $x^{-1} H=H$, so $x^{-1} \in H, H^{-1} \subset H$, and $H^{-1} H=H$. Thus $H$ is a (closed) subgroup of $G$. And since $x H$ and $H x$ must each be the set of constancy containing $x$, for any $x$ in $G, x H=H x, x \in G$, and $H$ is normal.

Let $\rho: G \rightarrow G / H$ be the canonical homomorphism, $\tilde{\rho}$ the induced isometry $f \rightarrow f \circ \rho$ of $C(G / H)$ into $C(G)$. Since $T$ commutes with translations, $T$ maps $\tilde{\rho} C(G / H)$ (the functions constant on cosets $\bmod H$ ) into itself: if $R_{h} f=f, h \in H$, then $T f(x h)=R_{h} T f(x)=T R_{h} f(x)=T f(x)$. Thus $T$ induces a bounded operator $T^{\prime}=\tilde{\rho}^{-1} T \tilde{\rho}$ on $C(G / H)$, which obviously acts as the identity on $A_{1}^{\prime}=\tilde{\rho} A_{1}$, an invariant separating subalgebra of $C(G / H)$. And $T^{\prime}$ commutes with translations and has range in $A_{2}^{\prime}=\tilde{\rho}^{-1}\left(A_{2} \cap \tilde{\rho} C(G / H)\right)$, an invariant subalgebra of $C(G / H)$ containing $A_{1}^{\prime}$ but not $\bar{A}_{1}^{\prime}$. So clearly it will suffice to obtain a contradiction from the existence of an operator $T$ commuting with translations, acting as the identity on $A_{1}$ and with range in $A_{2}$, just in the case that $A_{1}$ separates our group, and we shall henceforth assume $A_{1}$ separates $G$ so that, as we observed, $G$ is the Silov boundary of $A_{1}$.

Now the $A_{i}$, as invariant subspaces of $C(G)$, are spanned by minimal finite dimensional invariant subspaces $L$, and each $L$ has as a basis the entries in a finite dimensional unitary matricial representation $U$ of $G$. Moreover for any such $U$ either every entry of $U$ lies in $A_{i}$ or all lie outside $A_{i}$ (since the corresponding $L$ has $L \cap A_{i}=L$ or $\left.\{0\}\right)$. Consequently we shall speak of $U$ being "in" $A_{i}$ or "outside" $A_{i}$. (If $U$ is reducible we may still use these terms, though of course we have no dichotomy.)

Let us write $T U$ for the matrix $\left(T u_{i j}\right)$ of functions $\left(U=\left(u_{i j}\right)\right)$. Clearly, for any constant matrix $V$, we have $T V U=V T U, T U V=(T U) V$. Since $T$ commutes with translations, $T U(x)=R_{x} T U(1)=T R_{x} U(1)=T\left(U U_{x}\right)(1)$ $=\left[(T U) U_{x}\right](1)=(T U(1)) U_{x}$, and similarly $T U(x)=L_{x^{-1}} T U(1)=U_{x}(T U(1))$, so

$$
T U=V U=U V, \quad V=T U(1) .
$$

With $U$ irreducible, $V$ must be a scalar multiple of the identity, so $T U=\lambda U$, $\lambda$ complex. Hence

$$
T U=U \text { for } U \text { in } A_{1}
$$

since $T$ acts as the identity on $A_{1}$, while

$$
T U=0 \text { for } U \text { outside } A_{2}
$$


since the range of $T$ is contained in $A_{2}$. (Of course (3.3) and (3.4) remain valid if $U$ is reducible, but we do not need this.)

We shall next produce a nonzero invariant $\bar{A}_{1}$-module $M$ with $T M=0$. In order to do so we shall apply a generalized Stone-Weierstrass theorem obtained recently by Bishop $[3,6]$. Call $K \subset G$ a set of antisymmetry for $A_{2}$ if $f \in A_{2}$ and $f \mid K$ real-valued imply $f \mid K$ is constant. Then [6] the maximal sets of antisymmetry $K$ for $A_{2}$ form a closed pairwise disjoint covering $\mathscr{K}$ of $G$ for which

$$
f \in C(G) \text { and } f\left|K \in A_{2}\right| K \text { for all } K \text { in } \mathscr{K} \text { imply } f \in A_{2} .
$$

Now since the elements of $\mathscr{K}$ are maximal sets of antisymmetry, any translation maps an element of $\mathscr{K}$ onto another. Thus, as in our earlier consideration of sets of constancy, $\mathscr{K}$ consists of the cosets of a (closed, normal) subgroup (again called $H$ ) of $G$.

Now let $M$ be the closed span of the entries in all conjugates $\bar{U}$ of irreducible finite dimensional unitary (matrical) representations $U$ in $A_{1}$ with $\bar{U}$ outside $A_{2}$. Of course $M$ is invariant, and nonzero since $\bar{A}_{1} \ddagger A_{2}$, while $T M=0$ by (3.4).

In all that follows let $U, U^{\prime}$ be irreducible and in $A_{1}$. In order to show $M$ an $\bar{A}_{1}$-module it will suffice to show that for such $U, U^{\prime}$ in $A_{1}$, if $\bar{U}$ is outside $A_{2}$ then the conjugate $\overline{U \otimes U^{\prime}}$ of the tensor product $U \otimes U^{\prime}$ is outside $A_{2}$ as well. For $A_{1}$ is spanned by just the entries of such $U^{\prime}$, and the entries of $U \otimes U^{\prime}$ are just the products $u_{i j} u_{k l}^{\prime}$.

Recall that the maximal antisymmetric sets for $A_{2}$ are the cosets mod $H$. If $U \mid H$ contains the trivial representation then replacing $U$ by an equivalent representation we have

$$
U_{h}=\left(\begin{array}{ll}
1 & 0 \\
0 & V_{h}
\end{array}\right), \quad h \in H,
$$

so $u_{11}$ is constant on each coset of $H$. Since the same is then true of $\bar{u}_{11}, \bar{u}_{11} \in A_{2}$ by (3.5), whence $\bar{U}$ is in $A_{2}$. On the other hand if $\bar{U}$ is in $A_{2}$ then $U \mid H$ is constant since $H$ is a set of antisymmetry of $A_{2}$. So

(3.6) $\bar{U}$ is in $A_{2}$ if and only if $U \mid H$ contains the trivial representation.

Now let $U, U^{\prime}$ be in $A_{1}, \bar{U}$ outside $A_{2}$, and suppose $\overline{U \otimes U^{\prime}}$ is not outside $A_{2}$. Then some irreducible subrepresentation $V$ of $\overline{U \otimes U^{\prime}}$ is in $A_{2}$; since $U \otimes U^{\prime}$ is in $A_{1} \subset A_{2}$ and $H$ is a set of antisymmetry for $A_{2}, V \mid H$ is constant. Let

$$
U\left|H=\Sigma V_{i}, \quad U^{\prime}\right| H=\Sigma V_{j}^{\prime}
$$

represent the decomposition of $U \mid H$ and $U^{\prime} \mid H$ into irreducible unitary representations of $H$, so we may write $\left(U \otimes U^{\prime}\right) \mid H=\Sigma V_{i} \otimes V_{j}^{\prime}$, and $V_{i}, V_{j}^{\prime}$ are in $A_{2} \mid H$. Then for some $i, j$, some irreducible subrepresentation of $V_{i} \otimes V_{j}^{\prime}$ is constant, so $V_{i} \otimes V_{j}^{\prime}$ contains the trivial representation. Consequently [9, p. 438] $\bar{V}$ is unitarily equivalent to $V_{j}^{\prime}$, so $V_{i}$ and $\bar{V}_{i}$ are in $A_{2} \mid H$, hence constant. Thus 
$U \mid H$ contains the trivial representation and $\bar{U}$ is in $A_{2}$ by (3.6), contradicting the fact that $\bar{U}$ is outside $A_{2}$, so our proof that $M$ is an $A_{1}$-module is complete.

We now have the following situation: $G$ is the Šilov boundary of each of the algebras $A_{1}, \bar{A}_{1} ; A_{1}$ is an $A_{1}$-module without common zeroes on which the bounded operator $T$ acts as the identity, while $M$ is a nonzero $\bar{A}_{1}$-module with $T M=0$ and no common zeroes (since the set of common zeroes, being invariant, is either all of $G$ or void). This is precisely the situation excluded by 2.2 , so we have the desired contradiction and 3.1 is proved.

For $A_{1}=A_{2}=A$ nonselfadjoint and invariant, we thus have $A$ uncomplemented. On the other hand if $A$ is selfadjoint, the sets of constancy of $A$ are the cosets modulo a closed normal subgroup $H$, so $A$ consists of all functions constant on cosets mod $H$ (by Stone-Weierstrass). Consequently convolution with the normalized Haar measure of $H$ projects $C(G)$ onto $A$, and we have

Corollary 3.2. Let $A$ be a closed invariant subalgebra of $C(G), G$ compact. Then $A$ is complemented in $C(G)$ if and only if $A$ is selfadjoint.

It should be noted that to obtain our contradiction in the argument of 3.1 $P$ only had to be defined on an invariant subspace of $C(G)$ containing $A_{2}$ and $\bar{A}_{1}$. A consequence of this is the following.

COROLlaRY 3.3. Let $A$ be a closed invariant nonselfadjoint subalgebra of $C(G)$, with maximal ideal space $\mathscr{M}$. Let $A^{\wedge}$ denote the set of Gelfand representatives of $A$ in $C(\mathscr{M})$. Then $A^{\wedge}$ is uncomplemented in $C(\mathscr{M})$.

(Cf. the last part of 2.4; note that here the representing measures for elements of $\mathscr{M}$ need not be unique.)

Proof. Let $E$ be the subspace of $C(\mathscr{M})$ formed by all $f$ having $f(m)=\int f d \mu$ for all non-negative measures $\mu$ (on the Silov boundary of $A$ ) representing $m$, for each $m$ in $\mathscr{M}$. Then $E$ contains $A^{\wedge}$ and is selfadjoint.

As before the sets of constancy of $A$ are cosets mod $H$, for some normal subgroup $H$ of $G$, and we can identify $G / H$ as the Šilov boundary of $A$. Each translation (as an automorphism on $A$ ) induces a self homeomorphism of $\mathscr{M}$ which of course appears on the boundary $G / H$ as a translation by an element of $G$, and one easily sees that $E$ is invariant under these homeomorphisms. Since by definition

$$
|f(m)| \leqq \sup |f(G / H)|, \quad f \in E,
$$

$E$ is isometrically isomorphic to a closed subspace $E_{1}=(E \mid(G / H)) \circ \rho$ of $C(G)$, where $\rho: G \rightarrow G / H$ is the canonical map; and $E_{1}$ is a closed selfadjoint invariant subspace of $C(G)$ containing $A$, hence $\bar{A}$.

Consequently, a bounded projection of $C(\mathscr{M})$ onto $A$ (or just one of $E$ onto $A^{\wedge}$ ) induces a bounded projection of $E_{1}$ onto $A$; by our earlier observation the argument of 3.1 now applies (with $A_{1}=A=A_{2}$ ) to yield a contradiction. 
The proof of 3.1 makes essential use of the hypothesis that $A_{2}$ is an algebra, rather than just an $A_{1}$-module. The fact that $A_{2}$ can be just an $A_{1}$-module when $G$ is compact abelian yields a corresponding strengthening in that case of the following corollary $\left({ }^{6}\right)$, which contains Newman's result $[10,12,8]$ that the Hardy class $H_{1}$ is uncomplemented in $L_{1}$ of the circle.

Corollary 3.4. Let $G$ be a compact group, and let $A_{1} \subset A_{2}$ be invariant subalgebras of $C(G)$ with $\bar{A}_{1} \ddagger A_{2}$. Then any closed subspace $F$ of $L_{1}(G)$ lying between the $L_{1}$-closures of $A_{1}$ and $A_{2}$ is uncomplemented in $L_{1}(G)$.

Let $P$ be a bounded projection of $L_{1}(G)$ onto $F$. Since elements of $L_{1}(G)$ translate continuously, exactly as in 3.1 we may form the strongly convergent integrals

$$
\begin{aligned}
T_{1} f & =\int R_{x^{-1}} P R_{x} f d x, \\
T f & =\int L_{x^{-1}} T_{1} L_{x} f d x, \quad f \in L_{1}(G),
\end{aligned}
$$

obtaining a bounded operator $T$ on $L_{1}(G)$ commuting with translations, acting as the identity on the $L_{1}$-closure of $A_{1}$, and with range in the $L_{1}$-closure of $A_{2}$.

Now the adjoint $T^{*}$ on $L_{\infty}(G)$ also commutes with translations, and, as a consequence, $T^{*}$ leaves the subspace (formed by equivalence classes of elements of) $C(G)$ of $L_{\infty}(G)$ invariant; for this subspace consists of just the elements $f$ of $L_{\infty}(G)$ for which $x \rightarrow R_{x} f$ is a strongly continuous map into $L_{\infty}(G)$ (as appears from (4.3) below, for example). For $f \in C(G)$ then $T^{*} f(1)$ is well defined, and $f \rightarrow T^{*} f(1)$ is a bounded linear functional on $C(G)$; so we have a finite measure $\mu$ on $G$ for which $T^{*} f(1)=\int f(x) \mu(d x), f \in C(G)$.

But $T^{*} f(y)=R_{y} T^{*} f(1)=T^{*} R_{y} f(1)=\int f(x y) \mu(d x)$, and thus for $h \in L_{1}(G), \quad f \in C(G)$, we have $\int h(y) T^{*} f(y) d y=\iint h(y) f(x y) d y \mu(d x)$ $=\int\left(\int h\left(x^{-1} y\right) \mu(d x)\right) f(y) d y$, so

$$
T h(y)=\int h\left(x^{-1} y\right) \mu(d x)
$$

since $C(G)$ is weak* dense in $L_{\infty}(G)$. In particular (3.7) shows that the subspace $C(G)$ of $L_{1}(G)$ is invariant, and that the restriction $T_{0}$ of $T$ to $C(G)$ defines a bounded operator on $C(G)$.

Now if $T_{0} f \notin A_{2}$ for some $f \in C(G)$ we have $\left(T_{0} f\right) * h \notin A_{2}$ for some continuous function $h$ taken from an approximate identity; thus for some finite measure $v$ on $G$ orthogonal to $A_{2}$ we have

$$
0 \neq \int\left(T_{0} f\right) * h(x) v(d x)=\int\left(T_{0} f\right)(h * v) d x=\int(T f)(h * v) d x,
$$

(6) The author is indebted to the referee for this version of the result, which is considerably stronger than the original. 
and since $h * v$ is an element of $L_{\infty}(G)$ orthogonal to $A_{2}$, hence to the range of $T$, we have a contradiction. Consequently, $T_{0}$ has range in $A_{2}$.

Trivially $T_{0} f=f$ for $f$ in $A_{1}$, since this equality holds in $L_{1}(G)$, and so $T_{0}$ is a bounded operator on $C(G)$ acting as the identity on $A_{1}$, with range in $A_{2}$, and commuting with translations; since this is precisely the situation precluded by the proof of 3.1 (with $T=T_{0}$ ), we have the desired contradiction.

COROLlaRY 3.5. Let $G$ be a compact group, $A$ an invariant subalgebra of $C(G)$. Then the $L_{1}$-closure of $A$ is complemented in $L_{1}(G)$ if and only if $A$ is selfadjoint.

("If" follows as in 3.2.)

4. Let $G$ now be a locally compact abelian group. The present section is devoted to a characterization (implicit in [12] for $G$ compact) of the complemented invariant subspaces of $C_{0}(G)$ due to $\mathrm{K}$. deLeeuw, and to an analogue of 3.1 which follows from the same approach.

deLeeuw observed that an invariant mean on the space $m(G)$ of all bounded functions $\left({ }^{7}\right)$ on $G$ could be used in place of the Haar integral in (3.1), and had the following consequence.

TheOREM 4.1 (DELEeUW). A translation invariant subspace $A$ of $C_{0}(G)$ is complemented in $C_{0}(G)$ if and only if $A=\mu * C_{0}(G)$ for some idempotent measure $\mu=\mu * \mu$ on $G$. Consequently if $G$ has a connected dual $G^{\wedge}$, then no proper nonzero invariant subspace of $C_{0}(G)$ is complemented.

Proof. If $A=\mu * C_{0}(G), \mu=\mu * \mu$ then $f \rightarrow \mu * f$ provides a bounded projection of $C_{0}(G)$ onto $A$. Conversely let $P$ be a bounded projection onto $A$, and let us use $f \rightarrow M_{x} f(x)$ to denote our invariant mean on $m(G)$. Let $\langle\cdot, \cdot\rangle$ denote the usual pairing between $L_{\infty}(G)$ and $L_{1}(G):\langle f, h\rangle=\int_{G} f h d x, f \in L_{\infty}(G), \quad h \in L_{1}(G)$. For $f \in C_{0}(G)$, view $R_{-x} P R_{x} f$ as an element of $L_{\infty}(G)$ and form

$$
M_{x}\left\langle R_{-x} P R_{x} f, h\right\rangle \text { for } h \in L_{1}(G) .
$$

Trivially (4.1) is bounded by $\left\|P R_{x} f\right\|_{\infty}\|h\|_{1} \leqq\|P\|\|f\|_{\infty}\|h\|_{1}$, so we have an element $T f$ of $L_{\infty}(G)$ satisfying

$$
\langle T f, h\rangle=M_{x}\left\langle R_{-x} P R_{x} f, h\right\rangle, h \in L_{1}(G),
$$

and $T$ appears as a bounded map of $C_{0}(G)$ into $L_{\infty}(G)$.

Now by virtue of the invariance of our mean one easily obtains $T R_{x}=R_{x} T$; and this implies $T f$ translates continuously in $L_{\infty}(G)$ :

$$
\left\|R_{y} T f-T f\right\|_{\infty}=\left\|T\left(R_{y} f-f\right)\right\|_{\infty} \leqq\|T\|\left\|R_{y} f-f\right\|_{\infty}
$$

which tends to zero as $y$ approaches the identity 0 of $G$.

(7) That is, a non-negative functional (of norm 1) which is 1 at 1 and assumes the same value at $R_{x} f$ and $f, x \in G, f \in m(G)$. Such a mean always exists if $G$ is abelian. 
As a consequence $T f$ is (the equivalence class of) an element of $C(G)$. For $T f$ can be approximated (in the norm of $L_{\infty}$ ) by elements

$$
\int_{G} v(y) R_{-y} T f d y=v * T f
$$

of $L_{\infty}(G)$, where $v$ runs over an approximate identity in $L_{1}(G)$; since (4.3) is in fact in $C(G)$ the convergence of $\{v * T f\}$ in the essential supremum norm shows $\{v * T f\}$ is a Cauchy net in $C(G)$, whence our assertion.

So now we may view $T$ as a bounded map of $C_{0}(G)$ into $C(G)$, and $f \rightarrow T f(0)$ is a well-defined bounded linear functional on $C_{0}(G)$. Hence $T f(0)=\int f d \mu$, $f \in C_{0}(G)$, for some finite measure $\mu$ on $G$; and since $T f(x)=R_{x} T f(0)=T R_{x} f(0)$ $=\int R_{x} f d \mu=\mu * f(x)$,

$$
T f=\mu * f, \quad f \in C_{0}(G) .
$$

$\mu$ is finite, so this shows $T$ has its range in $C_{0}(G)$,. In fact $T f=f, f \in A$, while $T$ has its range in $A$, as we shall now verify.

For the first assertion, suppose $f \in A$ and $h \in L_{1}(G)$. Then since $R_{x} f \in A$, $P R_{x} f=R_{x} f$, so that

$$
\langle T f, h\rangle=M_{x}\left\langle R_{-x} P R_{x} f, h\right\rangle=M_{x}\langle f, h\rangle=\langle f, h\rangle,
$$

and $T f=f$ in $L_{\infty}(G)$, hence in $C_{0}(G)$. For the second assertion, note that if $f \in C_{0}(G)$ and $T f \notin A$ there is a finite measure $v$ orthogonal to $A$ for which $0 \neq \int T f d v=T f * v(0)$, whence $\int R_{x} T f d v \neq 0$ for $x$ near 0 in $G$. Thus for some $h \in L_{1}(G), 0 \neq\langle T f, v * h\rangle=\iint T f(y+x) v(d y) h(x) d x$, so that

$$
0 \neq M_{x}\left\langle R_{-x} P R_{x} f, v * h\right\rangle
$$

despite the fact that $v * h$ is an element of $L_{1}(G)$ orthogonal to $A$ (since $A$ is invariant) and $R_{-x} P R_{x} f \in A$ for each $x$ in $G$, whence

our contradiction.

$$
M_{x}\left\langle R_{-x} P R_{x} f, v * h\right\rangle=0,
$$

Now since $T f \in A$ for all $f$ in $C_{0}(G)$ while $T f=f$ for $f$ in $A, T$ is a projection onto $A$. Clearly $T^{2}=T$ implies $\mu * \mu=\mu$ by (4.4), and since $A$ is the range of $T, A=\mu * C_{0}(G)$ by (4.4).

For the final assertion of 4.1 , recall that when $G^{\wedge}$ is connected the only idempotents $\mu$ on $G$ are the zero measure and the unit mass at 0 (for the FourierStieltjes transform $\hat{\mu}=\hat{\mu}^{2}$ is identically zero or identically one). Thus $C_{0}(G)$ and $\{0\}$ are the only complemented invariant subspaces of $C_{0}(G)$.

The same approach, along with some facts from the spectral theory of bounded functions (see [7]), leads to our analogue of 3.1. For a closed subset $E$ of $G^{\wedge}$ let $k E$ be the ideal of $L_{1}(G)$ consisting of all $f$ with Fourier transform $\hat{f}$ vanishing on $E$, and let $J E$ be the closure of the ideal of $f$ in $L_{1}(G)$ with $f$ having compact 
support disjoint from $E$; thus $k E$ and $J E$ are, respectively, the largest and smallest closed ideals in $L_{1}(G)$ having $E$ as hull. Now let $C(G)_{\beta}$ denote the space of bounded continuous functions on $G$ endowed with the strict topology $[4] ; C(G)_{\beta}$ is a locally convex space whose dual can be identified with the space of finite measures on $G$, i.e., with $C_{0}(G)^{*}$ (as a vector space). $(k E)^{\perp}$ and $(J E)^{\perp}$ will denote the closed [7] subspaces of $C(G)_{\beta}$ orthogonal to $k E$ and $J E$, respectively; $(k E)^{\perp}$ is the (strictly) closed span of the elements of $E$ in $C(G)_{\beta}$, while $(J E)^{\perp}$ consists of all bounded continuous $f$ with spectrum $\operatorname{sp} f \subset E$.

We shall need one consequence of the fact that the set of continuous functionals on $C(G)_{\beta}$ coincides with those on $C_{0}(G)$ : if $B$ is a closed subspace of $C_{0}(G)$ then $B$ is a relatively closed subset of $C_{0}(G)$ in $C(G)_{\beta}$. For $B$ is weakly closed in $C_{0}(G)$, hence relatively closed in $C_{0}(G)$ in the weak topology of $C(G)_{\beta}$, and trivially this implies our assertion.

THEOREM 4.2. Let $G$ be a locally compact abelian group, $A$ a closed invariant subalgebra of $C_{0}(G)$, and $B$ an invariant $A$-module in $C_{0}(G)$ with $A \subset B, \bar{A} \ddagger B$. Then any closed subspace of $C_{0}(G)$ lying between $A$ and $B$ is uncomplemented in $C_{0}(G)$.

Proof. Again let $P$ be a bounded projection onto the subspace, and form $T$ as in (4.3). Exactly as before (i) $R_{x} T=T R_{x}$, and we obtain (ii) $T f=\mu * f, f \in C_{0}(G)$, and also the fact that (iii) $T f=f$ for $f$ in $A$. Moreover a simple adaptation of the argument showing $T$ in (4.3) had its range in $A$ shows that here (iv) $T$ has its range in $B$.

Now it suffices to obtain a contradiction from (i)-(iv) assuming $A$ separates $G$. For in general the sets of constancy of $A$ are cosets modulo a closed subgroup $H$ of $G$, as in 3.1, and $H$ is necessarily compact since $A \subset C_{0}(G)$. Hence if $\rho$ is the canonical map of $G$ onto $G / H$, and $\tilde{\rho}$ the induced map of $C_{0}(G / H)$ into $C_{0}(G)$, one easily obtains (i)-(iv) for $T_{1}=\tilde{\rho}^{-1} T \tilde{\rho}, A_{1}=\tilde{\rho}^{-1} A$ and $B_{1}=\tilde{\rho}^{-1} B$ (with (ii) replaced by its easy consequence $\tilde{\rho}^{-1} T \tilde{\rho} f=\left(\tilde{\rho}^{-1^{*}} \mu\right) * f, f \in C_{0}(G / H)$; thus our contradiction for the separating case will apply. Consequently we shall henceforth assume that $A$ separates $G$, hence that $G$ is the Šilov boundary of $A$.

With $\operatorname{sp} f$ the spectrum of $f \in C_{0}(G)$ and $E \subset C_{0}(G)$, let $\operatorname{sp} E=\left(\bigcup_{f \in E} \operatorname{sp} f\right)^{-}$. By [5, Theorem 4.7] $\operatorname{sp} A$ is a subsemigroup of $G^{\wedge}$, and by just the proof of that result one has $\operatorname{sp} A+\operatorname{sp} B \subset \operatorname{sp} B$ (since $A B \subset B$ and $A$ and $B$ are invariant).

Now we assert that $-\operatorname{sp} A \notin \operatorname{sp} B$. For suppose not, so that

$$
\operatorname{sp} A-\operatorname{sp} A \subset \operatorname{sp} A+\operatorname{sp} B \subset \operatorname{sp} B,
$$

and $\operatorname{sp} B$ contains the closure $g$ of the group $\operatorname{sp} A-\operatorname{sp} A$. Since $\operatorname{sp} A$ is a semigroup, $\operatorname{sp} A \subset g$ and $-\operatorname{sp} A \subset g$; thus among the elements of $(J g)^{\perp}$ (those $f$ in $\mathrm{C}(G)_{\beta}$ with $\operatorname{sp} f \subset g$ ) we have all $f$ in $A$, and all $\bar{f}$ in $\bar{A}$ (since $\operatorname{sp} \bar{f}=-\operatorname{sp} f$ ). Because $\operatorname{sp} f_{1} f_{2} \subset\left(\operatorname{sp} f_{1}+\operatorname{sp} f_{2}\right)^{-}[5, \S 3]$ while $g$ is a group, $(J g)^{\perp}$ contains the separating subalgebra of $C_{0}(G)$ generated by $A$ and $\bar{A}$, which is dense in $C_{0}(G)$ by Stone- 
Weierstrass. The injection of $C_{0}(G)$ into $C(G)_{\beta}$ is (obviously) continuous, so $(J g)^{\perp}$ contains a subset of $C_{0}(G)$ which is dense in $C_{0}(G)$ in the strict topology, and since $C_{0}(G)$ is dense in $C(G)_{\beta}$ while $(J g)^{\perp}$ is closed in $C(G)_{\beta}$, we thus have $(J g)^{\perp}=C(G)_{\beta}$. This of course implies $g=G^{\wedge}$, whence $\operatorname{sp} B=G^{\wedge}$, and $\bigcup_{f \in B} \operatorname{sp} f$ is dense in $G^{\hat{1}}$.

But each element of the dense subset $\bigcup_{f \in B} \operatorname{sp} f$ of $G^{\wedge}$ lies in the strictly closed span $F$ of $B$ [7, Theorem 1.2], and (since the injection of $G^{\wedge}$ into $C(G)_{\beta}$ is continuous) we thus have its closure $G^{\wedge} \subset F$. So $F$ contains the closed span of $G^{\wedge}$ in $C(G)_{\beta}$, i.e., $F \supset C(G)_{\beta}$. As we have noted, $B$ is relatively closed in $C_{0}(G)$ in the strict topology, so $B=F \cap C_{0}(G)=C_{0}(G)$, contradicting the fact that $A \nsubseteq B$. Hence $-\operatorname{sp} A \ddagger \operatorname{sp} B$ as asserted, or $\operatorname{sp} \bar{A} \ddagger \operatorname{sp} B$.

Now let $f_{1}$ be an element of $\bar{A}$ with $\operatorname{sp} f_{1} \ddagger \operatorname{sp} B$. Choosing an $h$ in $L_{1}(G)$ with Fourier transform $\hat{h}$ nonzero at some point of $\operatorname{sp} f_{1}$, yet having compact support disjoint from $\mathrm{sp} B$, we have $f_{2}=f_{1} * h$ a nonzero element of the (closed invariant) space $\bar{A}$, while $K=\operatorname{sp} f_{2}$ is a compact set disjoint from $\mathrm{sp} B$. Consequently $f_{2}^{2}$ is a nonzero element of $\bar{A}$ with spectrum contained in $K-\operatorname{sp} A$, and thus

$$
M=\bar{A} \cap(J(K-\operatorname{sp} A))^{\perp}=\{f: f \in A, \operatorname{sp} f \subset K-\operatorname{sp} A\} \neq\{0\} .
$$

Clearly $M$ is a relatively (strictly) closed subspace of $\bar{A}$ in $C(G)_{\beta}$, hence a closed subspace of $C_{0}(G)$ since the injection of $C_{0}(G)$ into $C(G)_{\beta}$ is continuous. Trivially $M$ is invariant, and, since $\operatorname{sp} f g \subset(\operatorname{sp} f+\operatorname{sp} g)^{-}[5, \S 3]$, for $f$ in $M$ and $g$ in $A$ we have

$$
\operatorname{sp} f g \subset K-\operatorname{sp} A-\operatorname{sp} A=K-\operatorname{sp} A
$$

since $\operatorname{sp} A$ is a closed semigroup and $K-\operatorname{sp} A$ is closed. Thus $f g \in M$, and $M$ is a (nonzero invariant) $\bar{A}$-module.

Now sp $M(\subset K-\operatorname{sp} A)$ and $\operatorname{sp} B$ are disjoint; for otherwise $\varnothing \neq(K-\operatorname{sp} A) \cap \operatorname{sp} B$, and $\varnothing \neq K \cap(\operatorname{sp} A+\operatorname{sp} B) \subset K \cap \operatorname{sp} B=\varnothing$. Recalling that $T f=\mu * f, f \in C_{0}(G)$, we have $\operatorname{sp} T f \subset \operatorname{sp} f$, and thus if $f \in M, \operatorname{sp} T f \subset K-\operatorname{sp} A$. Since $T f \in B, \operatorname{sp} T f=\varnothing$, and $T f=0$, for $f \in M$.

So we have precisely the situation encountered in $3.1: A$ and $\bar{A}$ have $G$ as their Silov boundaries, $A$ (resp., $M$ ) is an $A$-(resp., $\bar{A}$-) module with no common zeroes on $G$, while $T$ acts as the identity on $A$ and annihilates $M$, contradicting 2.2. Our proof of 4.2 is complete.

Exactly as in the compact case we have

Corollary 4.3. If $A$ is a closed invariant subalgebra of $C_{0}(G), G$ locally compact abelian, then $A$ is complemented in $C_{0}(G)$ if and only is $A$ is selfadjoint.

5. There is a slight strengthening of 2.1 in the case that $T$ is a projection and $F=C_{0}(X)$.

THEOREM 5.1. Let $X$ be the Šilov boundary of the closed subalgebra $A$ of 
$C_{0}(X)$ and let $B \subset C_{0}(X)$ be a proper closed $A$-module without common zeroes on $X$. Then any projection $P$ of $C_{0}(X)$ onto $B$ has norm $>2$.

Proof. We shall assume $\|P\| \leqq 2$ and obtain a contradiction. Take $F=C_{0}(X)$, $T=P$ in 2.1 . Then by $(2.1)$

$$
f(x)-P f(x)=\int f d v_{x}, f \in C_{0}(X)
$$

and by (2.2) (which holds for a dense set of $x$, hence by continuity for all $x$ )

$$
|f(x)-P f(x)| \leqq\|f\|(\|P\|-1) \leqq\|f\|, f \in C_{0}(X),
$$

so that $\left\|v_{x}\right\| \leqq 1$ for all $x$ in $X$. Consequently $x \rightarrow v_{x}$ is a weak* continuous (by (5.1)) map of $X$ into the unit ball of measures on $X$, in fact into the set $B^{\perp}$ of measures orthogonal to $B$.

Now

$$
P f \in B \text {, all } f \text { in } C_{0}(X),
$$

is equivalent to the fact that

$$
0=\int P f d \mu, \quad f \in C_{0}(X), \quad \mu \in B^{\perp}
$$

hence by (5.1) to

$$
0=\int\left[f(x)-v_{x}(f)\right] \mu(d x), \quad f \in C_{0}(X), \quad \mu \in B^{\perp},
$$

where $v_{x}(f)=\int f d v_{x}$. Consequently for $\mu$ in $B^{\perp}$,

$$
\int f d \mu=\int v_{x}(f) \mu(d x), \quad f \in C_{0}(X),
$$

and we have the representation

$$
\mu=\int v_{x} \mu(d x)
$$

of $\mu$ in $B^{\perp}$ as a weak* convergent integral.

Let $\mathscr{E}$ be the set of extreme points of ball $B^{\perp}=\left\{\mu: \mu\right.$ in $\left.B^{\perp},\|\mu\| \leqq 1\right\}$, which is nonvoid by the Krein-Milman theorem (since $B^{\perp} \neq \varnothing$ inasmuch as $B \neq C_{0}(X)$ ). Let $\mu \in \mathscr{E}$, so $\|\mu\|=1$, and let $K$ be any Borel set for which $|\mu|(K) \neq 0$, where $|\mu|$ is the total variation measure associated with $\mu$. Then $\left({ }^{8}\right)$

$$
\mu=\frac{1}{|\mu|(K)} \int_{K} v_{x} \mu(d x) .
$$

(8) Clearly (5.5) must imply $v_{x}$ is a (complex) multiple of $\mu \in \mathscr{E}$ for each $x$ in the carrier of $\mu$, but the proof of this fact will take some argument. 
(Here, of course, the integral is the measure corresponding to the well-defined continuous functional $f \rightarrow \int_{\mathrm{K}} v_{x}(f) \mu(d x)$ on $C_{0}(X)$.) Indeed, we have

$$
\mu=\int_{K} v_{x} \mu(d x)+\int_{\mathbb{R}^{\prime}} v_{x} \mu(d x)
$$

where each summand lies in $B^{\perp}$ since every $v_{x}$ does; since $\left\|v_{x}\right\| \leqq 1$,

$$
\begin{aligned}
\|\mu\| & \leqq\left\|\int_{K} v_{x} \mu(d x)\right\|+\left\|\int_{K^{\prime}} v_{x} \mu(d x)\right\| \\
& \leqq|\mu|(K)+|\mu|\left(K^{\prime}\right)=\|\mu\|=1
\end{aligned}
$$

so that equality holds throughout (5.6), and

$$
|\mu|(K)=\left\|\int_{K} v_{x} \mu(d x)\right\|, \quad|\mu|\left(K^{\prime}\right)=\left\|\int_{K^{\prime}} v_{x} \mu(d x)\right\| .
$$

Consequently (5.5) follows from the extremity of $\mu$.

Now let $\rho \mu=|\mu|$ where $\rho$ is a Borel function of modulus 1. By Lusin's theorem for $\eta>0$ there is a compact set $K$ for which the restriction $\rho \mid K$ is continuous, while $|\mu|(K)>1-\eta$. Let $\mu_{K}$ denote the restriction of $\mu$ to $K$, and carr $\mu_{K}$ the (closed) carrier of $\mu_{K}$, a subset of $K$. Then for $f \in C_{0}(X)$ and $x_{0} \in \operatorname{carr} \mu_{K}$ we have

$$
\left|v_{x}(f)-\frac{\rho(x)}{\rho\left(x_{0}\right)} v_{x_{0}}(f)\right|<\varepsilon
$$

for $x$ in $V \cap K$, where $V$ is some neighborhood of $x_{0}$; since $x_{0} \in \operatorname{carr} \mu_{K}$, $\left|\mu_{K}\right|(V)=|\mu|(K \cap V) \neq 0$. So by (5.5)

$$
\begin{aligned}
& \left|\mu(f)-\frac{v_{x_{0}}(f)}{\rho\left(x_{0}\right)}\right| \\
& =\left|\frac{1}{|\mu|(K \cap V)} \int_{K \cap V} v_{x}(f) \mu(d x)-\frac{1}{|\mu|(K \cap V)} \int_{K \cap V} \frac{v_{x_{0}}(f)}{\rho\left(x_{0}\right)} \rho(x) \mu(d x)\right|
\end{aligned}
$$

since $\int_{K \cap V} \rho(x) \mu(d x)=\int_{K \cap V}|\mu|(d x)=|\mu|(K \cap V)$, and

$$
\left|\mu(f)-\frac{v_{x_{0}}(f)}{\rho\left(x_{0}\right)}\right| \leqq \frac{1}{|\mu|(K \cap V)} \int_{K \cap V}\left|v_{x}(f)-\frac{\rho(x)}{\rho\left(x_{0}\right)} v_{x_{0}}(f)\right||\mu|(d x)<\varepsilon .
$$

Hence $v_{x_{0}}=\rho\left(x_{0}\right) \mu$ for $x_{0}$ in carr $\mu_{K}$, with $K$ as above.

Now the set of all such $x_{0}$ is necessarily dense in carr $\mu$. For if $x \in \operatorname{carr} \mu$ has a neighborhood $V$ for which $V \cap \operatorname{carr} \mu_{K}=\varnothing$ for all such $K$ then

$$
\left\|\mu_{\mathrm{K}}\right\| \leqq\|\mu\|-|\mu|(V)=1-|\mu|(V),
$$

while $\left\|\mu_{K}\right\|$ can be chosen arbitrarily close to 1 and $|\mu|(V)>0$. Thus $v_{x} \in T^{1} \mu, T^{1}$ the unimodular complex numbers, for a dense set of $x$ in carr $\mu$, 
hence for all $x$ in carr $\mu$ since $x \rightarrow v_{x}$ is weak* continuous and $T^{1} \mu$ is weak* closed.

Consequently if $\mu_{1}$ and $\mu_{2}$ are in $\mathscr{E}$, and their carriers meet, then $\mu_{1} \in T^{1} \mu_{2}$ since $t_{1} \mu_{1}=v_{x}=t_{2} \mu_{2}$ for some $x$.

Now fix $\mu \in \mathscr{E}$ and let $E=\left\{v: v \in\right.$ ball $B^{\perp}$, carr $v \subset$ carr $\left.\mu\right\}$, a weak* compact convex set. Then if $v$ is extreme in $E$ (so $\|v\|=1$ ), $v \in \mathscr{E}:$ for if

$$
v=a \lambda+(1-a) \lambda^{\prime}, \lambda, \lambda^{\prime} \in \text { ball } B^{\perp}, \quad 0<a<1
$$

and $\rho v=|v|$, where $\rho$ is a unimodular Borel function, then

$$
1=v(\rho)=a \rho \lambda(1)+(1-a) \rho \lambda^{\prime}(1)
$$

whence $1=\rho \lambda(1)=\rho \lambda^{\prime}(1) \geqq\|\rho \lambda\|,\left\|\rho \lambda^{\prime}\right\|$, and $\rho \lambda, \rho \lambda^{\prime}$ are non-negative. Trivially then carr $\lambda=\operatorname{carr} \rho \lambda$ and carr $\lambda^{\prime}=\operatorname{carr} \rho \lambda^{\prime}$ are contained in carr $\mu$, whence $\lambda, \lambda^{\prime}$ are in $E$, so that $v=\lambda=\lambda^{\prime}$ since $v$ is extreme in $E$.

From the fact that each extreme point $v$ of $E$ lies in $\mathscr{E}$ and has its carrier contained in carr $\mu$, we thus have $v \in T^{1} \mu$ for each such $v$, so that $E=D \mu$, where $D=\{z:|z| \leqq 1\}$. But since $B$ has no common zeroes, no element of $B^{\perp}$ (in particular $\mu$ ) is a point mass, so we have an element $f$ of $A$ which is nonconstant on carr $\mu$. So $f \mu \neq 0$ and $f \mu /\|f \mu\| \in E$, despite the fact that $f \mu /\|f \mu\| \in D \mu$ implies $f$ is constant on carr $\mu$; thus we have the desired contradiction, completing our proof.

\section{REFERENCES}

1. E. Bishop, A minimal boundary for function algebras, Pacific J. Math. 8 (1958), 629-642.

2. E. Bishop and $\mathrm{K}$. deLeeuw, The representation of linear functionals by measures on sets of extreme points, Ann. Inst. Fourier (Grenoble) 9 (1959), 305-331.

3. E. Bishop, A generalization of the Stone-Weierstrass theorem, Pacific J. Math. 11 (1961), 777-783.

4. R. C. Buck, Bounded continuous functions on a locally compact space, Michigan Math. J. 5 (1958), 95-104.

5. K. deLeeuw and H. Mirkil, Translation-invariant function algebras on abelian groups, Bull. Soc. Math. France 88 (1960), 345-370.

6. I. Glicksberg, Measures orthogonal to algebras and sets of antisymmetry, Trans. Amer. Math. Soc. 105 (1962), 415-435.

7. C. S. Herz, The spectral theory of bounded functions, Trans. Amer. Math. Soc. 94 (1960), 181-232.

8. K. Hoffman, Banach spaces of analytic functions, Prentice-Hall, Englewood Cliffs, N. J., 1962.

9. M. A. Naımark, Normed rings, Noordhoff, Groningen, 1959.

10. D. J. Newman, The non-existence of projections from $L^{1}$ to $H^{1}$, Proc. Amer. Math. Soc. 12 (1961), 98-99.

11. C. E. Rickart, General theory of Banach algebras, Van Nostrand, Princeton, N. J., 1960.

12. W. Rudin, Projections on invariant subspaces, Proc. Amer. Math. Soc. 13 (1962), 429-432.

UNIVERSITY OF WASHINGTON,

SeATtLe, Washington 